\title{
Minimally invasive thymectomy for myasthenia gravis: a 7-year retrospective study
}

\author{
Jian Gao ${ }^{1 \#}$, Chun Jin ${ }^{1,2 \#}$, Yong-Qiang Ao ${ }^{1}$, Jie Tang ${ }^{3}$, Jian-Yong Ding ${ }^{1}$, Ji-Hong Dong ${ }^{4}$, Jia-Hao Jiang ${ }^{1}$ \\ ${ }^{1}$ Department of Thoracic Surgery, Zhongshan Hospital, Fudan University, Shanghai, China; ${ }^{2}$ Department of Thoracic Surgery, Changhai hospital, \\ Naval Military Medical University, Shanghai, China; ${ }^{3}$ Department of Anesthesiology, Zhongshan Hospital, Fudan University, Shanghai, China; \\ ${ }^{4}$ Department of Neurology, Zhongshan Hospital, Fudan University, Shanghai, China \\ Contributions: (I) Conception and design: J Gao, JH Jiang, JH Dong; (II) Administrative support: JH Jiang; (III) Provision of study materials or \\ patients: C Jin, J Tang, YQ Ao, JY Ding; (IV) Collection and assembly of data: J Gao, C Jin; (V) Data analysis and interpretation: JH Jiang, JH Dong, \\ J Gao, C Jin, JY Ding; (VI) Manuscript writing: All authors; (VII) Final approval of manuscript: All authors. \\ \#These authors contributed equally to this work. \\ Correspondence to: Jia-Hao Jiang. Department of Thoracic Surgery, Zhongshan Hospital, Fudan University, 180 Fenglin Road, Shanghai 200032, \\ China. Email: jiang.jiahao@zs-hospital.sh.cn; Ji-Hong Dong. Department of Neurology, Zhongshan Hospital, Fudan University, 180 Fenglin Road, \\ Shanghai 200032, China. Email: dong.jihong@zs-hospital.sh.cn.
}

Background: Thymectomy has been identified as an effective strategy for patients with myasthenia gravis (MG) and thymic masses. However, the best surgical approach remains a matter of debate. The aim of the present study was to compare the surgical and neurological outcomes of video-assisted thoracoscopic thymectomy with a modified subxiphoid and bilateral approach in patients with MG and thymic masses.

Methods: From August 2013 to April 2018, 68 patients who were diagnosed with MG and thymic masses and underwent video-assisted thoracoscopic thymectomy with a modified subxiphoid (44 patients) or bilateral (24 patients) approach were included in this retrospective study. The surgical and neurological results were analyzed with propensity score matching.

Results: After propensity score matching, the modified subxiphoid approach in video-assisted thoracoscopic thymectomy resulted in an obviously shorter operative time $(\mathrm{P}=0.00)$, drainage duration $(\mathrm{P}=0.00)$, less intraoperative blood loss $(\mathrm{P}=0.00)$, and shorter postoperative hospital stay $(\mathrm{P}=0.01)$. In terms of neurological outcomes, no significant difference was observed in the improvement in MG, with 2-year complete stable remission rates of $21.1 \%$ and $26.3 \%(\mathrm{P}=0.68)$ and 2-year pharmacological remission rates of $31.6 \%$ and $26.3 \%(\mathrm{P}=0.60)$ for the bilateral and subxiphoid approaches, respectively. Additionally, the approaches resulted in similar effects on the magnitudes of decrease in the prednisolone and pyridostigmine doses after 2 years, with average pyridostigmine dose reductions of $72.2 \%$ and $71.1 \%(\mathrm{P}=0.78)$ and average prednisolone reductions of $76.8 \%$ and $71.7 \%(\mathrm{P}=0.96)$ for the bilateral and subxiphoid approaches, respectively.

Conclusions: The modified subxiphoid approach was found to be superior to the bilateral approach in video-assisted thoracic surgery thymectomy in terms of the surgical outcomes and yielded similar neurological outcomes. Therefore, the modified subxiphoid approach is recommended as an alternative to the bilateral approach in the treatment of patients with MG and thymic masses.

Keywords: Myasthenia gravis (MG); thymic mass; video-assisted thoracic surgery (VATS); bilateral approach; subxiphoid approach

Submitted Oct 19, 2021. Accepted for publication Dec 15, 2021.

doi: $10.21037 / g s-21-756$

View this article at: https://dx.doi.org/10.21037/gs-21-756 


\section{Introduction}

Myasthenia gravis (MG) is an autoimmune disorder characterized by fluctuating muscle weakness and fatigue. The mechanism of this disease is the dysfunction of neuromuscular junctions (NMJs), which is caused by the autoantibodies, such as those that act against the acetylcholine receptor (AChR), muscle specific tyrosine kinase (MuSK), and/or other components of the postsynaptic membrane at NMJs. It is strongly associated with the thymus, in that thymic mass, such as thymomas and thymic hyperplasia, play a vital role in the pathogenesis of MG (1). Therefore, in the current guidelines, thymectomy is recommended as a valuable strategy for the treatment of MG in addition to conventional medications, such as anticholinesterase agents and immunosuppressive drugs (2). Previous studies have reported that the complete stable remission (CSR) rate ranges from $30 \%$ to $60 \%$ after thymectomy (3-5). Video-assisted thoracic surgery (VATS) has been widely used as an alternative to open thymectomy in the treatment of MG and thymic masses $(6,7)$. Surgical approaches in VATS thymectomy include subxiphoid, transcervical, and lateral intercostal approaches, which can be further divided into unilateral and bilateral approaches $(8,9)$. While the lateral intercostal approach is most frequently performed in the clinical setting, subxiphoid VATS thymectomy has been gradually accepted by surgeons, as it offers the advantages of convenience and a better view of the cervical and bilateral thoracic cavities. However, the minimally invasive surgical approach for the treatment of MG patients remains a matter of debate, and thoracic surgeons are still investigating which approach provides enough space for the precise resection of the thymus in the mediastinum and better recovery, prevents nerve injury and other complications, and achieves comparable or even better MG remission.

The aim of the present study was to compare the surgical and neurological outcomes of a modified subxiphoid and bilateral VATS thymectomy for patients with MG and thymic masses. We present the following article in accordance with the STROBE reporting checklist (available at https://dx.doi.org/10.21037/gs-21-756).

\section{Methods}

This retrospective study included patients who were diagnosed with MG and thymic masses and underwent VATS thymectomy from August 2013 to April 2018.
Relevant records, such as patient demographics, pathological results, and intraoperative and postoperative data, were retrieved from our database and reviewed. Patients with incomplete follow up or clinical data were excluded. The diagnosis of MG was based on clinical presentations, electromyographic findings, neostigmine test results, and detection results of autoantibodies acting against NMJs, such as AChR, MuSK, titin, and low-density lipoprotein receptorrelated protein antibodies. MG cases MG were classified according to the Myasthenia Gravis Foundation of America (MGFA) clinical classification system, and cases ranged from ocular muscle involvement to myasthenic crisis. The prognosis of these patients was evaluated with the MGFA postintervention status, and we mainly focused on CSR, defined as complete recovery without any treatment for at least 1 year, and pharmacological remission (PR), which is similar to CSR but involves treatment with medications other than cholinesterase inhibitors (10). Pathological results were assessed by 2 pathological experts, according the World Health Organization histological classification system (11). The clinical stage of thymoma was determined according to the Masaoka-Koga staging system (12). From August 2013 to December 2016, 24 patients underwent bilateral intercostal VATS thymectomy, and then 44 patients underwent VATS thymectomy with the modified subxiphoid approach. All patients underwent preoperative evaluations in a multidisciplinary manner, including neurological symptom assessments, electrodiagnosis, antibody tests, contrast-enhanced computed tomography, and cardiopulmonary function tests. Written informed consent was obtained from the patients, and ethical approval was obtained from the Zhongshan Hospital Research Ethics Committee (No. Y2019-187). All procedures performed in this study involving human participants were in accordance with the Declaration of Helsinki (as revised in 2013).

\section{Procedures for modified subxiphoid VATS thymectomy}

The patient was placed in the supine scissors position with a pad under the back to lift the chest. The surgeon stood at the middle of the patient. After intubation and sterilization, a 2- to $3-\mathrm{cm}$ observation port was made $1.5 \mathrm{~cm}$ under the lower edge of the xiphoid for thoracoscopy. Two 5 -mm working ports were located under the 2 -sided costal arch; these ports were approximately $5 \mathrm{~cm}$ away from the observation port and used for operation of the ultrasonic scalpel and thoracoscopic grasping forceps. The artificial $\mathrm{CO}_{2}$ pneumothorax pressure was set at 


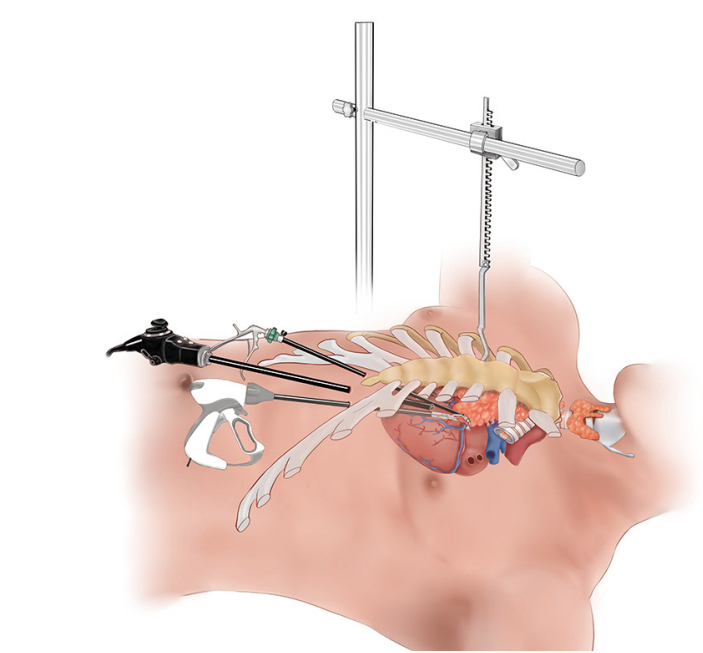

Figure 1 Subxiphoid approach in patients with myasthenia gravis and thymic masses.

$8 \mathrm{mmHg}$. The surgical procedures were as follows. First, the operator opened the retrosternal space with an ultrasonic scalpel to extend the operating view, and bluntly separated the bilateral cardiophrenic angle of the thymus and peripheral fatty tissues. The anterior border of the thymus was then separated along the retrosternal space to the thoracic outlet, and a small incision was made in the second intercostal parasternal space to insert the hook. The stretching height of the hook was determined according to the operating view and the judgment of the operator. Later, the mediastinal pleura was opened, and upward separation of the thymus and peripheral fatty tissues was performed to the innominate vein. During this process, the margins of bilateral separation were the phrenic nerves; injury to these 2 nerves should be avoided, especially in MG patients. Finally, the operator opened the upper mediastinal pleura and removed the cervical peritracheal fatty tissues as much as possible. The thymus was carefully separated along the surface of the innominate vein. After complete separation, the thymus was placed into a specimen bag and removed from the observation port, and the incision was closed. A negative-pressure drainage ball remained indwelling in the observation port for 2-3 days. A diagram of this approach is shown in Figure 1.

\section{Procedures for bilateral VATS thymectomy}

The bilateral VATS thymectomy approach has been previously reported (13). After intubation, the patient was placed in the semi-supine position with 60-degree retroversion to the right, and the arm was raised above the head. A 2- to 3-cm observation port was made in the 7 th intercostal space on the midaxillary line for thoracoscopy. Two 5-mm ports were located in the 5 th intercostal space on the midaxillary line and anterior axillary line for operation of the ultrasonic scalpel and thoracoscopic grasping forceps. The artificial $\mathrm{CO}_{2}$ pneumothorax pressure was also set at $8 \mathrm{mmHg}$. The thoracic adhesion was divided by the ultrasonic scalpel. The mediastinal pleura was opened between the sternum and the pericardium. The surgeon then began to separate the thymus and peripheral fatty tissues in the left cardiophrenic angle with an ultrasonic scalpel and cautiously continued dissection of the thymus along the phrenic nerve and the surface of the pericardium to the innominate vein. The surgeon carefully separated the thymus along the surface of the innominate vein as far as possible. A drain was placed in the thoracic cavity, and the incisions were closed. When the left approach was finished, the patient was placed into a semi-supine position with 45-degree retroversion to the left. An observation port was made in the 6th intercostal space on the midaxillary line. Another 2 working ports were separately introduced in the 5 th intercostal space on the anterior axillary line and the 3 rd intercostal space on the midaxillary line. The operator opened the mediastinal pleura between the sternum and the pericardium, and continued separation of the thymus and peripheral fatty tissues in the right cardiophrenic angle, with upward dissection along the phrenic nerve and the surface of pericardium to the innominate vein with the ultrasonic scalpel. The remaining thymus on the innominate vein was carefully separated. Both upper poles of the thymus were dissected cephalically as far as possible. A drain was placed in the thoracic cavity and the incisions were closed. Finally, a 5 -cm low-collar incision was made 2 fingerbreadths above the sternal notch to expose the upper pole of the thymus. All fatty tissues in front of the trachea were removed. The thymus was then removed via the subxiphoid incision.

\section{Statistical analysis}

Statistical analysis was performed with SPSS version 23.0 (IBM, Armonk, NY, USA) or R 3.5.1 statistical software (Boston, Massachusetts). Continuous variables are presented as the mean \pm standard deviation. Categorical variables were evaluated with the $\chi^{2}$-test. For continuous variables, Student's $t$-test was applied to compare normally distributed variables, and the Mann-Whitney U-test was 
used for nonnormally distributed variables. Propensity score matching analysis was performed to minimize the differences in preoperative variables between the 2 groups, and the scores were calculated by logistic regression, including the following variables: sex, age, pathology, Masaoka-Koga staging, Osserman classification, positive autoantibodies, abnormal repetitive nerve stimulation response, mean pyridostigmine and prednisolone dosage, and tumor size. The 1:1 nearest neighbor matching strategy was chosen to select the patients for the 2 groups. CSR and PR rates were measured according to the KaplanMeier method and log-rank test to evaluate the significant difference in MG remission between the 2 groups. All $\mathrm{P}$ values were 2 tailed, and $\mathrm{P}<0.05$ was regarded as statistically significant.

\section{Results}

\section{Surgical outcomes}

The clinicopathological characteristics are presented in Table 1. A total of 68 patients were diagnosed with MG and thymic masses. Of these, 24 underwent bilateral intercostal VATS thymectomy and 44 underwent VATS thymectomy with the modified subxiphoid approach between August 2013 and April 2018. Before propensity score matching, significant differences were observed between the 2 groups in terms of sex. After 1:1 matching, 19 patients in the bilateral group and 19 patients in the subxiphoid group were included. All variables were comparable between the 2 groups. Four patients had myasthenic crisis before surgery, and the symptoms gradually improved with highdose methylprednisolone or intravenous immunoglobulin, followed by prednisolone, anticholinesterase agent pyridostigmine, and mechanical ventilation.

Table 2 shows the surgical outcomes of patients who underwent bilateral and subxiphoid VATS thymectomy. No cases of perioperative or interoperative mortality or conversion to open thymectomy were recorded in the 2 groups. All patients underwent extended resection for complete removal of the thymus and fatty tissues. Before propensity score matching, the intraoperative outcomes of the subxiphoid group were significantly better than those of the bilateral group, with an operation time of $83 \pm 32.6$ and $165.6 \pm 43.5 \mathrm{~min}(\mathrm{P}=0.00)$ and blood loss of $25.7 \pm 18.9$ and $71.3 \pm 52 \mathrm{~mL}(\mathrm{P}=0.00)$, respectively. In terms of postoperative outcomes, the subxiphoid group had a shorter drainage duration $(2.2 \pm 1.8$ vs. $4 \pm 1.7$ days, $\mathrm{P}=0.00)$ and postoperative hospital stay $(5 \pm 1.9$ vs. $6 \pm 1.9$ days, $\mathrm{P}=0.02)$, while the intensive care unit (ICU) stay was similar in the 2 groups $(2.6 \pm 1.5$ vs. $3 \pm 1.7$ days, $\mathrm{P}=0.48)$. Both groups had 2 cases of postoperative complications, with 1 case of pneumonia and 1 case of myasthenic crisis in the bilateral group, and 2 cases of myasthenic crisis in the subxiphoid group; no significant difference was observed $(\mathrm{P}=0.53)$. After matching, the surgical outcomes were similar to the results of the whole cohort. The subxiphoid approach still achieved advantages in operation time, blood loss, drainage duration, and postoperative hospital stay. Therefore, the subxiphoid group had an obvious advantage over the bilateral group in terms of surgical outcomes.

\section{Postoperative follow up}

The last follow up was in November 2021, and the median follow-up duration was 48 months. No cases of relapse were identified before the end of the follow-up period. Most patients underwent neurological follow up at our hospital $(n=37)$. One patient died of heart failure 4 years after thymectomy, and 1 patient suffered from myasthenic crisis and was treated with mechanical ventilation and medication adjustment during follow up. The cumulative incidence of $M G$ remission after propensity score matching is presented in Figure 2. According to the Kaplan-Meier and log-rank analyses, the CSR rate was comparable between the 2 groups, with a 2 -year CSR rate of $21.1 \%$ in the bilateral group and $26.3 \%$ in the subxiphoid group $(\mathrm{P}=0.68)$ (Figure $2 A)$. In terms of the $\mathrm{PR}$ rate, there was no significant difference between the 2 groups, with a 2 -year PR rate of $31.6 \%$ in the bilateral group and $26.3 \%$ in the subxiphoid group $(\mathrm{P}=0.60)$ (Figure $2 B)$. Figure 3 shows the dose reduction in anticholinesterase agents and immunosuppressive drugs at 2 years post-thymectomy after propensity score matching. Both groups achieved similar medication dose reductions, corresponding with the results of CSR and PR. The 2-year pyridostigmine dose reduction was $71.1 \%$ in the bilateral group and $72.2 \%$ in the subxiphoid group $(\mathrm{P}=0.78)$ (Figure $3 A$ ), and the 2-year prednisolone dose reduction in the 2 groups was $76.8 \%$ and $71.7 \%$, respectively $(\mathrm{P}=0.96)$ (Figure $3 B)$.

\section{Discussion}

Given the existence of ectopic thymic tissues, complete resection has been the standard treatment for thymic masses, especially for patients with MG (14). Different 
Table 1 Clinical characteristics of patients who underwent bilateral and subxiphoid video-assisted thoracic surgery thymectomy before and after propensity score matching

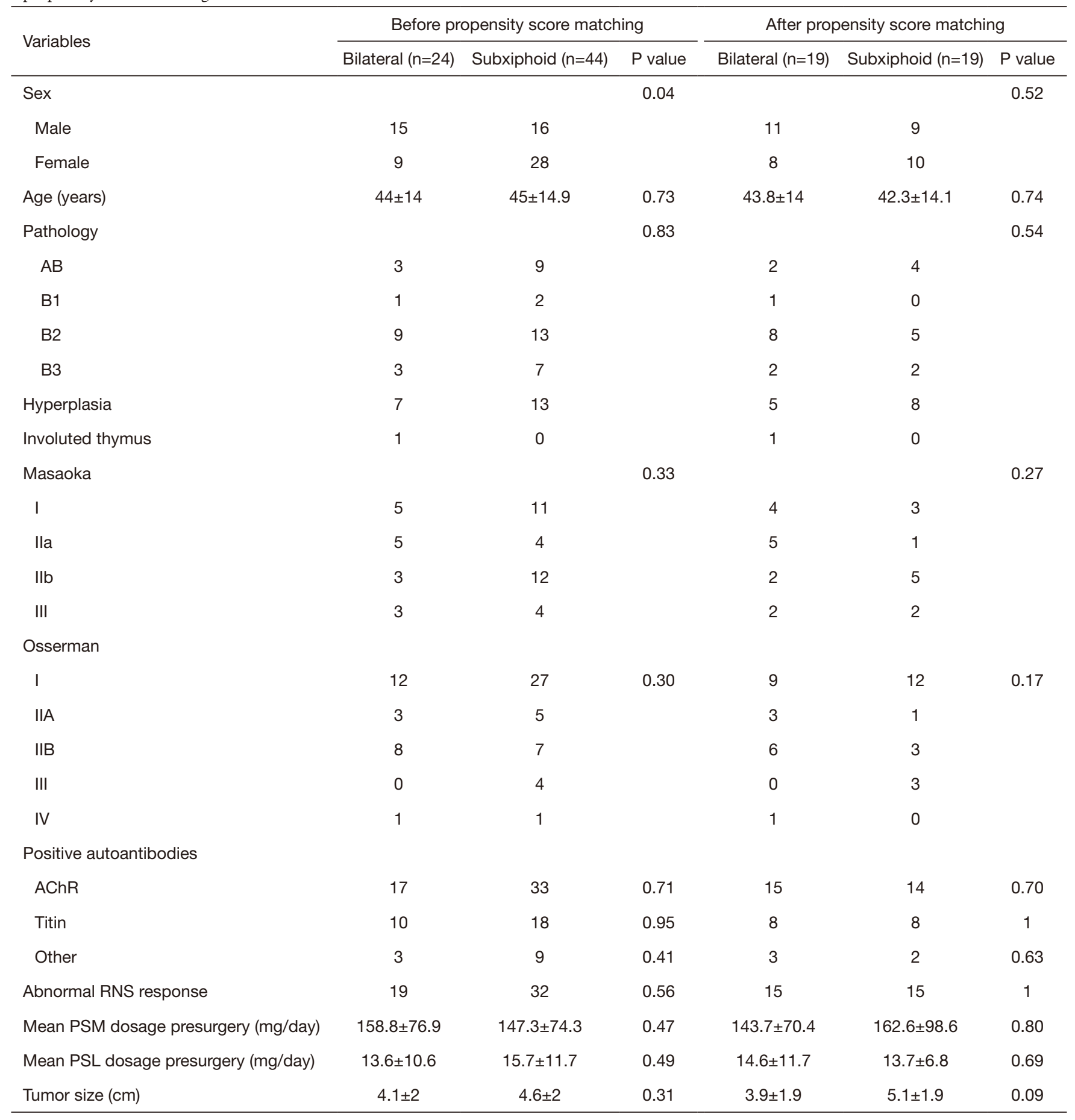

AChR, acetylcholine receptor; PSL, prednisolone; PSM, pyridostigmine; RNS, repetitive nerve stimulation. 
Table 2 Surgical outcomes of patients who underwent bilateral and subxiphoid video-assisted thoracic surgery thymectomy before and after propensity score matching

\begin{tabular}{|c|c|c|c|c|c|c|}
\hline Variables & \multicolumn{3}{|c|}{ Before propensity score matching } & \multicolumn{3}{|c|}{ After propensity score matching } \\
\hline Operation time (min) & $165.6 \pm 43.5$ & $83 \pm 32.6$ & 0.00 & $160 \pm 48.4$ & $78.9 \pm 17.2$ & 0.00 \\
\hline Drainage duration (day) & $4 \pm 1.7$ & $2.2 \pm 1.8$ & 0.00 & $3.7 \pm 1.5$ & $2 \pm 1.7$ & 0.00 \\
\hline Intraoperative blood loss $(\mathrm{mL})$ & $71.3 \pm 52$ & $25.7 \pm 18.9$ & 0.00 & $66.3 \pm 48.4$ & $29 \pm 16.3$ & 0.00 \\
\hline Postoperative hospital stay (days) & $6 \pm 1.9$ & $5 \pm 1.9$ & 0.02 & $5.8 \pm 1.8$ & $4.8 \pm 1.8$ & 0.01 \\
\hline Postoperative ICU stay (days) & $3 \pm 1.7$ & $2.6 \pm 1.5$ & 0.48 & $2.9 \pm 1.6$ & $2.6 \pm 1.6$ & 0.58 \\
\hline Postoperative complications & & & 0.53 & & & 0.31 \\
\hline Pneumonia & 1 & 0 & & 0 & 0 & \\
\hline
\end{tabular}

ICU, intensive care unit; VAS, visual analogue score.
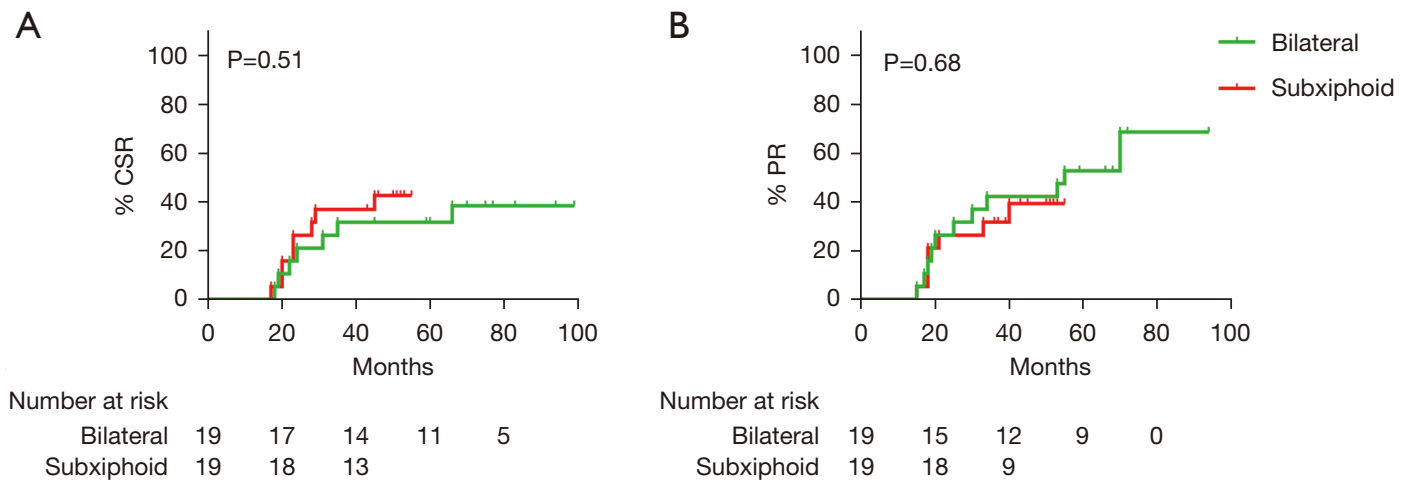

Figure 2 Clinical remission status of myasthenia gravis patients after propensity score matching. (A) Complete stable remission rate of patients who underwent bilateral and subxiphoid VATS thymectomy; (B) pharmacological remission rate of patients who underwent bilateral and subxiphoid VATS thymectomy. VATS, video-assisted thoracic surgery.

surgical approaches have been reported, including open and VATS thymectomy. Open thymectomy is still widely applied in the clinical setting due to its technical advantages, and previous studies have confirmed its superiority in oncological and neurological results. In recent years, VATS thymectomy has gained more attention in the treatment of patients with MG and thymic masses, as it also ensures the complete removal of thymic tissues and avoids the disadvantages of open thymectomy. The incision wounds of bilateral VATS thymectomy are smaller and less noticeable than those of the open approach. However, while the bilateral approach overcomes the disadvantage of potential contralateral phrenic nerve injury of the unilateral approach, it is more complicated and intercostal nerve injury is still inevitable. In contrast, these drawbacks can be solved by the subxiphoid approach, which provides a sufficient view of bilateral phrenic nerves and is not performed through the intercostal space $(15,16)$. Zhang et al. compared the subxiphoid with lateral intercostal approaches in early thymoma and found that patients in the subxiphoid approach group yielded lower pain scores, shorter postoperative hospital stays, decreased inflammatory cytokine response and superior cosmesis (17). Recently, other study reported that the robotic single-site-assisted 
A

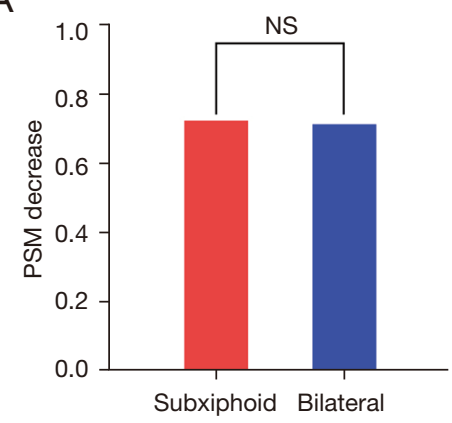

B

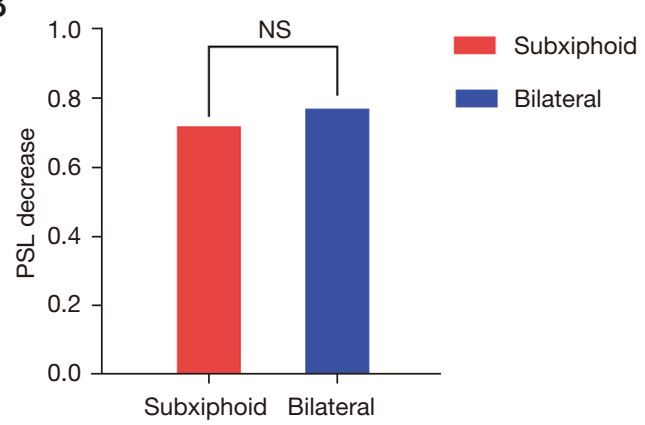

Figure 3 Dose reduction of anticholinesterase agents and immunosuppressive drugs at 2 years post-thymectomy after propensity score matching: (A) pyridostigmine dose reduction 2 years after bilateral and subxiphoid VATS thymectomy; (B) prednisolone dose reduction 2 years after bilateral and subxiphoid VATS thymectomy. NS, no significance; VATS, video-assisted thoracic surgery.

thymectomy via the subxiphoid approach is also a safe and technically feasible procedure (18). After the first report of subxiphoid approach for thymectomy by Hsu et al. in 2002, the subxiphoid thymectomy has gradually been accepted in the clinical setting (19). Nevertheless, as with other minimally invasive approaches, this approach also has the disadvantage of a limited operating space. Patients with large thymic masses or locally advanced thymomas might undergo conversion to the open approach. Therefore, we modified the subxiphoid approach by using a hook to lift the sternum, thereby providing a better surgical view, which is vital for resection in the mediastinum to be performed with high accuracy. In December 2016, the standard treatment for patients with MG and thymic masses was converted from the bilateral intercostal approach to the modified subxiphoid approach in VATS thymectomy at our center. In addition, we adopted the subxiphoid and subcostal arch approach, rather than the uniportal subxiphoid approach, as the surgical instruments interfered with each other in the single port in the latter approach.

A recent randomized controlled trial of thymectomy in patients with non-thymomatous MG revealed that thymectomy was a feasible treatment to improve the clinical outcomes in terms of a lower quantitative MG score and prednisolone dose compared with medical treatment alone (20). However, the surgical approach remains a matter of debate. Derderian et al. compared open and VATS thymectomy for juvenile MG and found that VATS thymectomy was a safe and effective approach (21). Hsu et al. compared the unilateral intercostal and subxiphoid bilateral approaches in the treatment of $\mathrm{MG}$, and the results revealed that while both approaches achieved comparable remission in $M G$, the subxiphoid approach provided a better surgical view (8). Recently, robotic thymectomy has gradually become popular, and many studies have reported that robotic thymectomy is safe and feasible for patients with MG, with a 5 -year CSR rate ranging from $28 \%$ to $42 \%$ and a low rate of intraoperative and postoperative complications (22-24).

In our retrospective study, we compared the bilateral intercostal approach and the modified subxiphoid approach in VATS thymectomy in terms of surgical and neurological results. The results showed the benefits of the modified subxiphoid approach in the treatment of patients with MG and thymic masses compared with the bilateral intercostal approach. After propensity score matching, the subxiphoid group showed a significantly lower mean operative duration and intraoperative blood loss. Drainage duration and postoperative hospital stay were also obviously reduced in the subxiphoid group, as only a negative-pressure absorbing ball was placed for drainage, and 7 patients did not even require drainage. In addition, the postoperative ICU stay and postoperative complications were similar between the 2 groups. Phrenic nerve injury is a severe complication that can result in a reduced expiratory volume and other postoperative complications, especially in patients with MG (25). Previous studies have reported intraoperative phrenic nerve injury in unilateral VATS thymectomy, as it is difficult to identify the contralateral nerve (17). However, there were no cases of phrenic nerve injury in the bilateral or subxiphoid group due to the advantage of precise identification of the bilateral phrenic nerve in the 2 approaches.

The follow-up MG classification system was recommended by the MGFA in 2000, and we applied this classification to evaluate the prognosis of MG after VATS thymectomy. We found similar neurological results with 
both approaches. No significant differences were observed between the 2 groups in terms of CSR or PR. The 2-year CSR rate was $21.1 \%$ in the bilateral group and $26.3 \%$ in the subxiphoid group. The 2-year PR rate was $31.6 \%$ in the bilateral group and $26.3 \%$ in the subxiphoid group. A previous study showed a pyridostigmine and prednisolone dose reduction of over $70 \% 2$ years after thymectomy (26). In the present study, both approaches also achieved similar reductions in anticholinesterase and immunosuppressive drug doses. The 2-year pyridostigmine dose reduction was $71.1 \%$ in the bilateral group and $72.2 \%$ in the subxiphoid group, and the 2-year prednisolone dose reduction in the 2 groups was $76.8 \%$ and $71.7 \%$, respectively. The neurological results suggested that both approaches are similarly effective in thymus resection and have high accuracy.

The present study has some limitations. First, the small sample size might not be sufficient to compare the 2 approaches in the treatment of patients with MG and thymic masses, and precludes further analysis of the 2 approaches for cases with different Osserman classifications. Second, the retrospective nature of the study could lead to selection bias. However, we performed propensity score matching analysis to ensure that the 2 groups were comparable. Finally, the postoperative follow-up duration for the subxiphoid approach was relatively short, and a longer observation time is needed for validation.

\section{Conclusions}

In the present study, we compared the surgical and neurological results of bilateral intercostal and subxiphoid approaches in the treatment of patients with $M G$ and thymic masses. The modified subxiphoid approach was found to be superior to the bilateral approach in VATS thymectomy in terms of surgical outcomes, and neurological outcomes were similar. Therefore, modified subxiphoid approach is recommended as an alternative to the bilateral approach in the treatment of patients with MG.

\section{Acknowledgments}

Funding: This study was funded by the Outstanding Youth Program of Zhongshan Hospital (2019ZSYXQN09).

\section{Footnote}

Reporting Checklist: The authors have completed the STROBE reporting checklist. Available at https://dx.doi. org/10.21037/gs-21-756

Data Sharing Statement: Available at https://dx.doi. org/10.21037/gs-21-756

Conflicts of Interest: All authors have completed the ICMJE uniform disclosure form (available at https://dx.doi. org/10.21037/gs-21-756). The authors have no conflicts of interest to declare.

Ethical Statement: The authors are accountable for all aspects of the work in ensuring that questions related to the accuracy or integrity of any part of the work are appropriately investigated and resolved. Written informed consent was obtained from the patients, and ethical approval was obtained from the Zhongshan Hospital Research Ethics Committee (No. Y2019-187). All procedures performed in this study involving human participants were in accordance with the Declaration of Helsinki (as revised in 2013).

Open Access Statement: This is an Open Access article distributed in accordance with the Creative Commons Attribution-NonCommercial-NoDerivs 4.0 International License (CC BY-NC-ND 4.0), which permits the noncommercial replication and distribution of the article with the strict proviso that no changes or edits are made and the original work is properly cited (including links to both the formal publication through the relevant DOI and the license). See: https://creativecommons.org/licenses/by-nc-nd/4.0/.

\section{References}

1. Meriggioli MN, Sanders DB. Autoimmune myasthenia gravis: emerging clinical and biological heterogeneity. Lancet Neurol 2009;8:475-90.

2. Gilhus NE, Verschuuren JJ. Myasthenia gravis: subgroup classification and therapeutic strategies. Lancet Neurol 2015;14:1023-36.

3. Popescu I, Tomulescu V, Ion V, et al. Thymectomy by thoracoscopic approach in myasthenia gravis. Surg Endosc 2002;16:679-84.

4. Savcenko M, Wendt GK, Prince SL, et al. Videoassisted thymectomy for myasthenia gravis: an update of a single institution experience. Eur J Cardiothorac Surg 2002;22:978-83.

5. Mantegazza R, Baggi F, Bernasconi P, et al. Videoassisted thoracoscopic extended thymectomy and extended transsternal thymectomy (T-3b) in non-thymomatous 
myasthenia gravis patients: remission after 6 years of follow-up. J Neurol Sci 2003;212:31-6.

6. Nakagiri T, Inoue M, Shintani $\mathrm{Y}$, et al. Improved procedures and comparative results for video-assisted thoracoscopic extended thymectomy for myasthenia gravis. Surg Endosc 2015;29:2859-65.

7. Raza A, Woo E. Video-assisted thoracoscopic surgery versus sternotomy in thymectomy for thymoma and myasthenia gravis. Ann Cardiothorac Surg 2016;5:33-7.

8. Qiu Z, Chen L, Lin Q, et al. Perioperative outcomes and mid-term effects in performing video-assisted thoracoscopic extended thymectomy for myasthenia gravis: subxiphoid versus right thoracic approaches. J Thorac Dis 2020;12:1529-39.

9. Migliore M, Alongi G, Rampello L, et al. Video assisted trans-cervical thymectomy: a minimally invasive approach to treat non-thymomatous myasthenia gravis. Ann Ital Chir 2013;84:667-70.

10. Jaretzki A 3rd, Barohn RJ, Ernstoff RM, et al. Myasthenia gravis: recommendations for clinical research standards. Task Force of the Medical Scientific Advisory Board of the Myasthenia Gravis Foundation of America. Ann Thorac Surg 2000;70:327-34.

11. Marx A, Chan JK, Coindre JM, et al. The 2015 World Health Organization Classification of Tumors of the Thymus: Continuity and Changes. J Thorac Oncol 2015;10:1383-95.

12. Detterbeck FC, Nicholson AG, Kondo K, et al. The Masaoka-Koga stage classification for thymic malignancies: clarification and definition of terms. J Thorac Oncol 2011;6:S1710-6.

13. Xue L, Pang X, Zhang Y, et al. Extended thymectomy by a cervical incision additional to bilateral VATS approach. J Vis Surg 2017;3:83.

14. Ambrogi V, Tacconi F, Sellitri F, et al. Subxiphoid completion thymectomy for refractory non-thymomatous myasthenia gravis. J Thorac Dis 2020;12:2388-94.

15. Numanami H, Yano M, Yamaji M, et al. Thoracoscopic Thymectomy Using a Subxiphoid Approach for Anterior Mediastinal Tumors. Ann Thorac Cardiovasc Surg

Cite this article as: Gao J, Jin C, Ao YQ, Tang J, Ding JY, Dong JH, Jiang JH. Minimally invasive thymectomy for myasthenia gravis: a 7 -year retrospective study. Gland Surg 2021;10(12):3342-3350. doi: 10.21037/gs-21-756
2018;24:65-72.

16. Suda T, Hachimaru A, Tochii D, et al. Video-assisted thoracoscopic thymectomy versus subxiphoid single-port thymectomy: initial results $\dagger$. Eur J Cardiothorac Surg 2016;49 Suppl 1:i54-8.

17. Zhang L, Li M, Jiang F, et al. Subxiphoid versus lateral intercostal approaches thoracoscopic thymectomy for non-myasthenic early-stage thymoma: A propensity score -matched analysis. Int J Surg 2019;67:13-7.

18. Park SY, Han KN, Hong JI, et al. Subxiphoid approach for robotic single-site-assisted thymectomy. Eur J Cardiothorac Surg 2020;58:i34-8.

19. Hsu CP, Chuang CY, Hsu NY, et al. Subxiphoid approach for video-assisted thoracoscopic extended thymectomy in treating myasthenia gravis. Interact Cardiovasc Thorac Surg 2002;1:4-8.

20. Wolfe GI, Kaminski HJ, Cutter GR. Randomized Trial of Thymectomy in Myasthenia Gravis. N Engl J Med 2016;375:2006-7.

21. Derderian SC, Potter DD, Bansal S, et al. Open versus thoracoscopic thymectomy for juvenile myasthenia gravis. J Pediatr Surg 2020;5 5:1850-3.

22. Rückert JC, Swierzy M, Ismail M. Comparison of robotic and nonrobotic thoracoscopic thymectomy: a cohort study. J Thorac Cardiovasc Surg 2011;141:673-7.

23. Freeman RK, Ascioti AJ, Van Woerkom JM, et al. Long-term follow-up after robotic thymectomy for nonthymomatous myasthenia gravis. Ann Thorac Surg 2011;92:1018-22; discussion 1022-3.

24. Keijzers M, de Baets M, Hochstenbag M, et al. Robotic thymectomy in patients with myasthenia gravis: neurological and surgical outcomes. Eur J Cardiothorac Surg 2015;48:40-5.

25. Yano M, Sasaki H, Moriyama S, et al. Preservation of phrenic nerve involved by stage III thymoma. Ann Thorac Surg 2010;89:1612-9.

26. Uzawa A, Kawaguchi N, Kanai T, et al. Two-year outcome of thymectomy in non-thymomatous late-onset myasthenia gravis. J Neurol 2015;262:1019-23. 